123 


\title{
Analysis of the Spike Rate and Spike Correlations in Modulated Semiconductor Lasers with Optical Feedback
}

\author{
Taciano Sorrentino, C. Quintero-Quiroz, M. C. Torrent, and Cristina Masoller
}

\begin{abstract}
We investigate experimentally how changes in the external cavity length and DC current affect the mean inter-spikeinterval (ISI) in a modulated semiconductor laser with optical feedback operating in the low-frequency fluctuations regime. The variation of the mean ISI with the modulation frequency is shown to be more pronounced when time delay and DC current allow for low spike rate. We use the method of ordinal symbolic analysis to examine how time correlations (among 3,4 and 5 consecutive laser spikes) change with the spike rate. This method is able to capture subtle changes, otherwise hidden in the dynamics. We find that higher spike rates wash out the effects of the modulation in the time correlations. Simulations using the Lang and Kobayashi model are in good qualitative agreement with the experimental observations.
\end{abstract}

Index Terms-Semiconductor Laser, Optical Feedback, Diode Laser Modulation, Optical Neuron, Low-Frequency Fluctuations, Excitability.

\section{INTRODUCTION}

$\mathbf{O}$ PTICAL feedback in semiconductor lasers has attracted a lot of attention in the last three decades both for being relevant to applications (detrimental in some cases, desirable in others) and for the rich dynamical behavior that it causes (see, for example, [1], [2] and references therein). One of the remarkable phenomena observed in semiconductor lasers with optical feedback is known as low-frequency fluctuations (LFFs) [3]-[13]. It is usually observed for moderate feedback levels when the injection current is near the solitary laser threshold. It can also be caused by current modulation when the laser is under optical feedback [14]. LFFs consist of dropouts of the laser output intensity, in an apparently random fashion, followed by gradual step-like recovery, with an average frequency that is much lower than the characteristic frequencies of the system, namely, the external cavity frequency and the relaxation oscillation frequency. The mean time interval between intensity dropouts is proportional to the reflectivity of the external reflector, proportional to the external cavity round-trip time, and inversely proportional to the injection current [3], [6].

T. Sorrentino is with the Departament de Física i Enginyeria Nuclear, Universitat Politècnica de Catalunya, Colom 11, 08222 Terrassa, Barcelona, Spain, and also with the Departamento de Ciências Exatas e Naturais, Universidade Federal Rural do Semi-Árido, Av. Francisco Mota 572, 59625900 Mossoró, RN, Brazil (e-mail: taciano@ufersa.edu.br).

C. Quintero-Quiroz, M. C. Torrent and C. Masoller are with the Departament de Física i Enginyeria Nuclear, Universitat Politècnica de Catalunya, Colom 11, 08222 Terrassa, Barcelona, Spain (e-mail: carlos.alberto.quintero@upc.edu; carme.torrent@upc.edu; cristina.masoller@upc.edu).

Manuscript received $\mathrm{xxxx} \mathrm{xx}, \mathrm{xxxx}$; revised $\mathrm{xxxxx} \mathrm{xx}, \mathrm{xxxx}$.
The LFF dynamics is often excitable [5], [7], [15]. As excitable behavior in neurons is known to be intimately related with information processing in the brain [16], the recent years saw many efforts to develop excitable photonic devices that could mimic neuronal activity in information processing networks inspired by biological systems. In particular, semiconductor lasers with optical injection [17]-[21], optical feedback [22]-[25], both [26], or saturated absorbers [27]-[30] have been investigated as possible optical spiking neurons.

In [24] we analyzed the spiking output of a semiconductor laser in the LFF regime using a symbolic method of timeseries analysis capable of detecting subtle variations in timecorrelated data and showed that, at the symbolic level, the laser dynamics can be well reproduced by a minimal model already known for describing time correlations in sensory neurons [31]. When the laser is subject to an external forcing through direct current modulation [14], [32]-[39], the minimal model also reproduces the symbolic dynamics. Those results suggest that LFF dropouts (from now on called spikes) can be used to simulate neuronal spikes in optical information processing schemes. As neuronal systems encode information in sequences of correlated spikes [40], [41], and temporal correlation may be used as information carrier, a relevant question is how temporal correlations among optical spikes are affected by variations in the spike rate imposed by the laser parameters.

Here we study experimentally the influence of the feedback delay time and of the DC value of the injection current, when the laser is current-modulated and the modulations frequency varies over a comprehensive range encompassing the average LFF frequency without modulation. For different external cavity lengths and DC currents we measured the mean interspike-interval (ISI) as a function of the modulation frequency. We find that the differences in the mean ISI for the different modulation frequencies can be large or small, depending on the laser parameters. We then use ordinal analysis [24], [25], [42] to investigate the effect of varying the spike rate in the spike correlations. We find that higher spike rates wash out the effect of the current modulation in the temporal correlations. Our findings suggest that the spike rates must be taken into account if one wants to exploit temporal correlations in modulated optical spikes to encode information. Simulations using the well-known Lang and Kobayashi model are in good qualitative agreement with the experimental observations. 


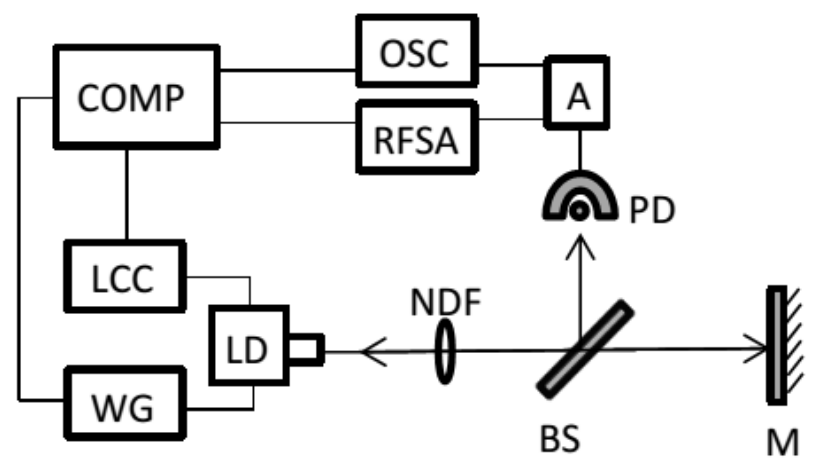

Fig. 1. Schematics of the experimental setup. LD: laser diode; NDF: neutral density filter, BS: beam-splitter; M: mirror; PD: photo-detector; A: fast amplifier; OSC: digital storage oscilloscope; RFSA: radio frequency spectrum analyzer; COMP: computer; LCC: laser combi controller; WG: waveform generator.

\section{EXPERIMENTAL SETUP}

The experimental setup is depicted in Fig. 1. A semiconductor laser (Sony SLD1137VS), with a solitary threshold current $I_{t h}=28.40 \mathrm{~mA}$, temperature- and current-stabilized with an accuracy of $0.01 \mathrm{C}$ and $0.01 \mathrm{~mA}$, respectively, using a diode laser combi controller (Thorlabs ITC501), emitting at $650 \mathrm{~nm}$, has part of its output power fed back to the laser cavity by a mirror. A 50/50 beamsplitter in the external cavity sends light to a photo-detector (Thorlabs DET210) that is connected to a fast amplifier (FEMTO HSA-Y-2-40), a $1 \mathrm{GHz}$ digital storage oscilloscope (Agilent Technologies Infiniium DSO9104A) and a radio frequency spectrum analyzer (Anritsu MS2651B). A neutral density filter in the external cavity allows to control the feedback power. The laser is operated at $17.00 \mathrm{C}$ and, unless stated, the threshold reduction due to feedback is $7.3 \%$. In the experiment we used three external cavity lengths, corresponding to feedback delay times, $\tau$, of 2.5, 5 and 7.5 $\mathrm{ns}$, and the DC current value was varied in the range between between $1.01 I_{t h}$ and $1.05 I_{t h}$.

A bias-tee in the laser mount allows the pump current to be modulated with a sinusoidal signal provided by a 80 $\mathrm{MHz}$ waveform generator (Agilent 33250A), with frequency varying from 1 to $50 \mathrm{MHz}$ in steps of $1 \mathrm{MHz}$, and peak-topeak amplitudes, $A_{\text {mod }}=0.8 \%$ and $1.6 \%$ of $I_{t h}$. Only for the higher modulation amplitude and the lower $I_{D C}$ the laser operates momentarily below the solitary threshold $I_{t h}$, in a range where the LFFs are still observed, and no remarkable qualitative difference due to this fact appears. For all other values of modulation amplitude and $I_{D C}$ the laser current is always above $I_{t h}$. The experiment is controlled by a LabVIEW program that acquires the time series, detects the spikes, and calculates the inter-spike-intervals (ISIs) until a minimum of 60,000 ISIs are recorded. Then, the program changes the modulation frequency and/or amplitude, waits 10 seconds to let transients die away, and the process is repeated.

\section{LANG AND KoBAYASHI MODEL}

The Lang and Kobayashi rate equations [43], in adimensional form, for the slowly varying complex electric field $E$ and the carrier density $N$ are

$$
\begin{gathered}
\frac{d E}{d t}=\frac{1}{2 \tau_{p}}(1+i \alpha)(G-1) E+\eta E(t-\tau) e^{-i \omega_{0} \tau}+\sqrt{2 \beta_{s p}} \xi \\
\frac{d N}{d t}=\frac{1}{\tau_{N}}\left(\mu-N-G|E|^{2}\right),
\end{gathered}
$$

where $\alpha$ is the linewidth enhacement factor, $\tau_{p}$ and $\tau_{N}$ are the photon and carrier lifetimes respectively, $G=N /(1+$ $\epsilon|E|^{2}$ ) is the optical gain (with $\epsilon$ a saturation coefficient), $\mu$ is the pump current parameter, $\eta$ is the feedback coupling coefficient, $\tau$ is the feedback delay time, $\omega_{0}$ is the solitary laser frequency, $\omega_{0} \tau$ is the feedback phase, $\beta_{s p}$ is the noise strength, representing spontaneous emission, and $\xi$ is a Gaussian distribution with zero mean and unit variance. The current modulation is simulated as $\mu=\mu_{0}+a \sin \left(2 \pi f_{\text {mod }} t\right)$, where $a$ is the modulation amplitude, $f_{\text {mod }}$ is the modulation frequency and $\mu_{0}$ is the DC current. We used in the simulations $a=0.004,0.008$, that correspond, respectively, to peak-topeak amplitudes $A_{\text {mod }}=0.8 \%$ and $1.6 \%$ of the threshold current, that is $\mu=1$ in this model. $f_{m o d}$ varies from 1 to 51 $\mathrm{MHz}$, in steps of $1 \mathrm{MHz}$.

The laser parameters used in the simulations are typical. In all simulations we used: $\epsilon=0.01, \tau_{p}=1.67 \mathrm{ps}, \tau_{N}=1$ $\mathrm{ns}, \beta_{s p}=5 \times 10^{-5} \mathrm{~ns}^{-1}, \eta=10 \mathrm{~ns}^{-1}$, and $\alpha=4$. To simulate the experimental situations we used three values of $\tau, \tau=2.5,5$ and $7.5 \mathrm{~ns}$, and $\mu_{0}$ is varied between 1.01 and 1.03. For each modulation frequency we simulated $2 \mathrm{~ms}$ and averaged the intensity time series over a sliding window of $1 \mathrm{~ns}$ to reproduce the bandwidth of the detection system. The averaged series contained between 21230 and 74680 ISIs, depending on the parameters.

\section{ORdinal Symbolic Analysis}

Ordinal analysis [44] and other advanced nonlinear tools have been recently used to investigate the nonlinear dynamics of semiconductor lasers [24], [25], [42], [45]-[49]. Here we analyze the simulated and the experimental ISI sequences using ordinal analysis, as in [24], [25], [42]. Each ISI sequence, $\left\{\Delta T_{i}\right\}$, is transformed into a sequence of ordinal patterns (OPs), which are defined by considering the relative length of $D$ consecutive ISIs and assigning them a symbol that indicates their relative length, in the same order as they appear in the sequence. The shortest interval is assigned 0 and the longest interval is assigned $D-1$. For $D=2$ the only two possibilities are: $\Delta T_{i}>\Delta T_{i+1}$ that gives the '10' $\mathrm{OP}$, and $\Delta T_{i}<\Delta T_{i+1}$ that gives the ' 01 ' OP. For $D=3$ there are six possibilities: $\Delta T_{i}<\Delta T_{i+1}<\Delta T_{i+2}$ gives '012', $\Delta T_{i+1}<\Delta T_{i}<\Delta T_{i+2}$ gives '102', and so on. As an example, in Fig. $2 \mathrm{~b}$ the ISIs forming an ' 102 ' $\mathrm{OP}$ are shown. For $D=4$ the OPs are defined similarly. The OPs probabilities are then calculated by counting their frequency of occurrence in the sequence.

This symbolic transformation has the drawback that it disregards the information about the precise duration of the ISIs, but it has the advantage that it keeps the information about temporal correlations among them, i.e., about correlations in the timing of the optical spikes. Specifically, in the section VI we analyze correlations among 3 spikes (by using $D=2$ 


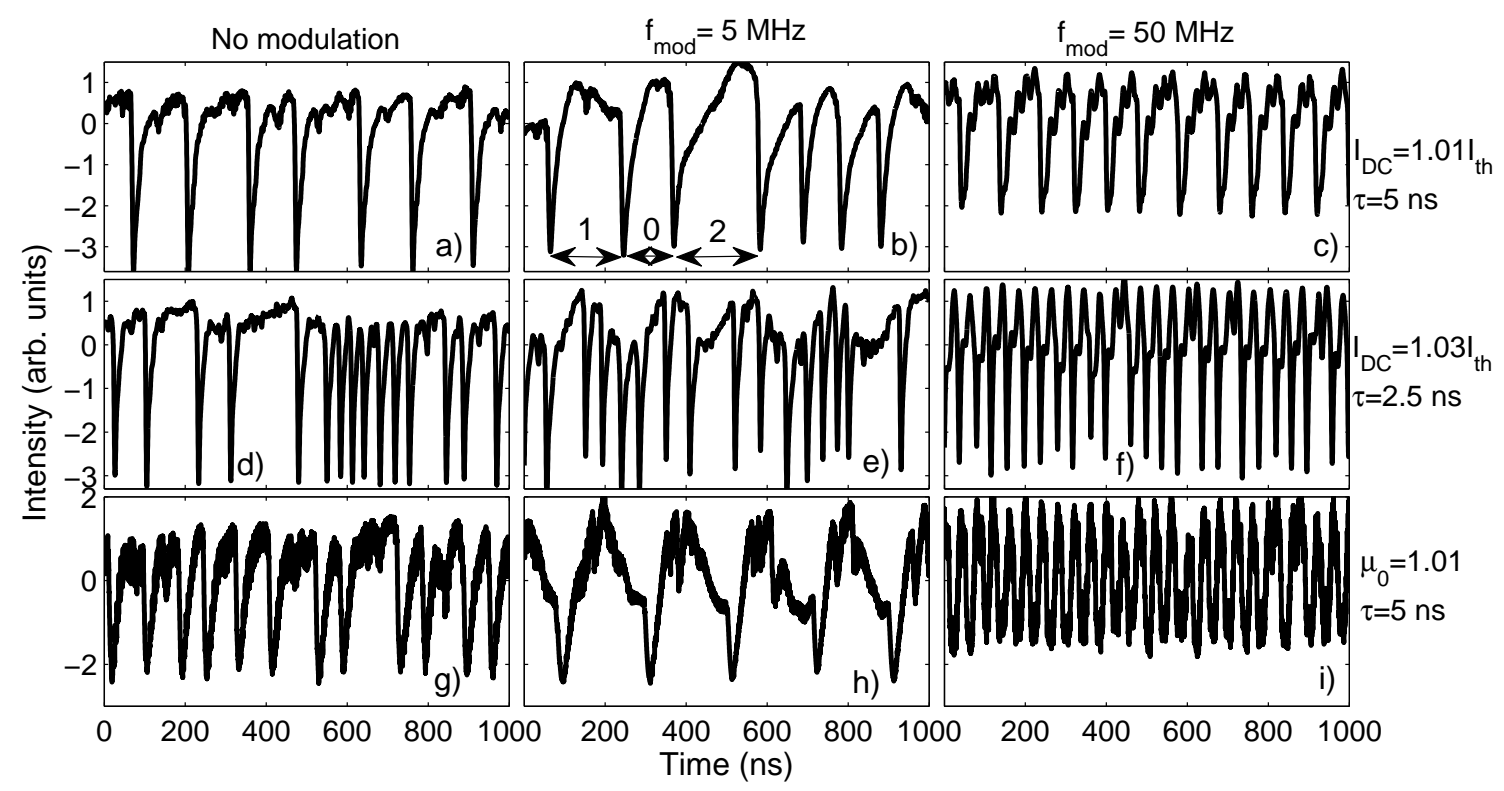

Fig. 2. Experimental and simulated intensity time series with and without modulation for different spike rate conditions. $A_{m o d}=1.6 \%$ of $I_{t h}$. a-f: experimental. g-i: simulations. a-c: $I_{D C}=1.01 I_{t h}, \tau=5$ ns. d-f: $I_{D C}=1.03 I_{t h}, \tau=2.5 \mathrm{~ns}$. g-i: $\mu_{0}=1.01, \tau=5$ ns. a,d,g: no modulation. b,e,h: $f_{m o d}=5$ MHz. $\mathrm{c}, \mathrm{f}, \mathrm{i}: f_{\text {mod }}=50 \mathrm{MHz}$.

OPs), 4 spikes (by using $D=3$ OPs) and 5 spikes (by using $D=4$ OPs).

\section{Analysis of the Spike Rate of the Modulated LASER}

Time series showing the lasers spikes for different conditions are displayed in Fig. 2. In all the panels the time interval of $1 \mu \mathrm{s}$, and the modulation amplitude is $1.6 \%$ of $I_{t h}$. The panels in the same line are for the same $\tau$ and $I_{D C}\left(\mu_{0}\right)$, the panels in the same column are for the same modulation frequency $f_{\text {mod }}$, or no modulation. In the panels $2 \mathrm{a}-\mathrm{c}$, where the parameters allow for a relatively slow LFF dynamics, we can see that a slow $f_{\text {mod }}(2 \mathrm{~b})$ do not change remarkably the spike rate present in the unmodulated laser $(2 a)$, while a fast $f_{\text {mod }}$ provokes a considerable increase in the spike rate as we can see in panel 2c, where the spikes are entrained: they occur each 3 or 4 modulation cycles. In the faster LFF dynamics of panels $2 \mathrm{e}-\mathrm{f}$ we also see that the slow $f_{\text {mod }}(2 \mathrm{e})$ does not change much the spike rate we have in the unmodulated case (2d). In $2 \mathrm{f}$ the dropouts are also entrained, occurring each 2 or 3 modulation cycles.

Panels 2g-h display time series simulated with the Lang and Kobayashi model, for $\mu_{0}=1.01$ and $\tau=5 \mathrm{~ns}$. The LFF dynamics is a bit faster in the simulations. Despite this fact, we shall see that the influence of $\tau$ and $I_{D C}$ in the spike rates and correlations observed in the experiments is qualitatively well reproduced by the model. We note a general qualitative agreement between panels $2 \mathrm{a}-\mathrm{c}$ and $2 \mathrm{~g}$-i. For fast $f_{\text {mod }}$, the dropouts in the numerical series are also entrained, occurring each 2 or 3 modulation cycles (panel 2i).

The effects of varying the time delay and the pump current on the experimental spike rate are shown in Fig. 3. The modulation amplitude is as in Fig. 2. In panel 3a the mean ISI for three external cavities, corresponding to time delays of $2.5,5$ and $7.5 \mathrm{~ns}$, are plotted against the modulation frequency. The curves for 5 and $7.5 \mathrm{~ns}$ present a plateau for low frequencies, followed by a rapid decrease in the mean ISIs as the modulation frequency increases, and a local minimum and maximum, after which the mean ISI varies little for 7.5 $\mathrm{ns}$, and continue to decrease for $5 \mathrm{~ns}$. The local minimum and maximum occur for higher frequencies in the curve for $5 \mathrm{~ns}$ and are absent in the curve for $2.5 \mathrm{~ns}$, where the mean ISI decrease almost monotonically. Varying the current, Fig. $3 \mathrm{~b}$, one can follow the variations in the spike rate in a more gradual way. In panel $3 \mathrm{~b}$ the curves for low $I_{D C}$ resemble the curves for $\tau=5,7.5 \mathrm{~ns}$ in 3a. As the current increases, the plateau in the low frequency region increases and local minimum and maximum move to higher frequencies, while the curves become more flat.

Figure 4 presents the results of simulations. The mean ISI vs. modulation frequency for different $\tau$ and $\mu_{0}$ is displayed. The numerical curves resemble the experimental ones, the plateau for low frequency and the following rapid decrease can be seen. The main difference is the oscillations that occur at intermediate and high frequencies, much stronger in the experimental curves. We can see a small oscillation in the curves for $\tau=5,7.5 \mathrm{~ns}$ in panel $4 \mathrm{a}$ and the curve for $\mu_{0}=1.01$ in panel $4 \mathrm{~b}$.

From Figs. 3 and 4 we can conclude that when the parameters are such that the natural spike rate (without modulation) is slow (i.e., for long delay or low $I_{D C}$ ) then, the modulation frequency affects more strongly the mean ISI, that, with exception of a few narrow intervals, decreases with increasing modulation frequency. In other words, faster modulation is able to produce faster spikes. On the contrary, when the spikes without modulation are already fast (for short delay or for large $\left.I_{D C}\right)$ then, the modulation frequency has a smaller effect in the spike rate. 


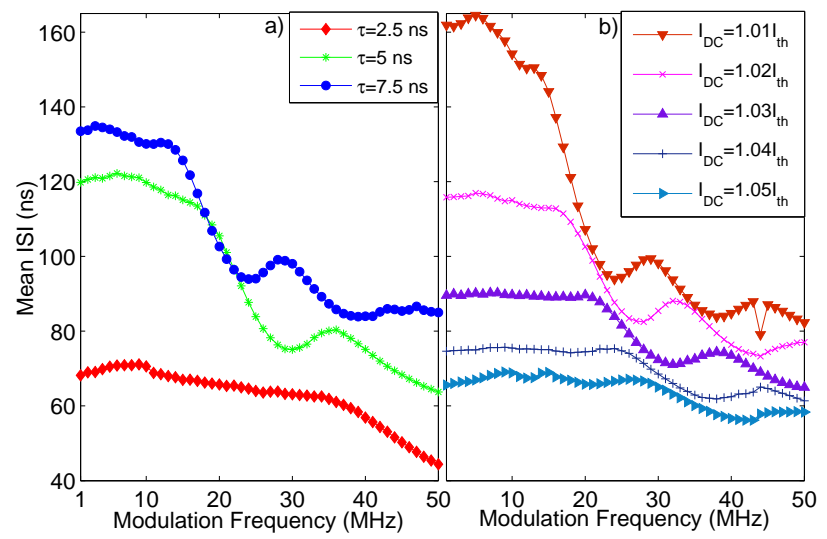

Fig. 3. a) Experimental mean ISI as function of the modulation frequency for three different time delays. 2.5 and $7.5 \mathrm{~ns}: I_{D C}=1.03 I_{t h} .5 \mathrm{~ns}: I_{D C}=$ $1.024 I_{t h}$, threshold reduction of $7.1 \%$. b) Experimental mean ISI as function of the modulation frequency for five different DC currents. $\tau=5 \mathrm{~ns} . A_{\bmod }=$ $1.6 \%$ of $I_{t h}$.

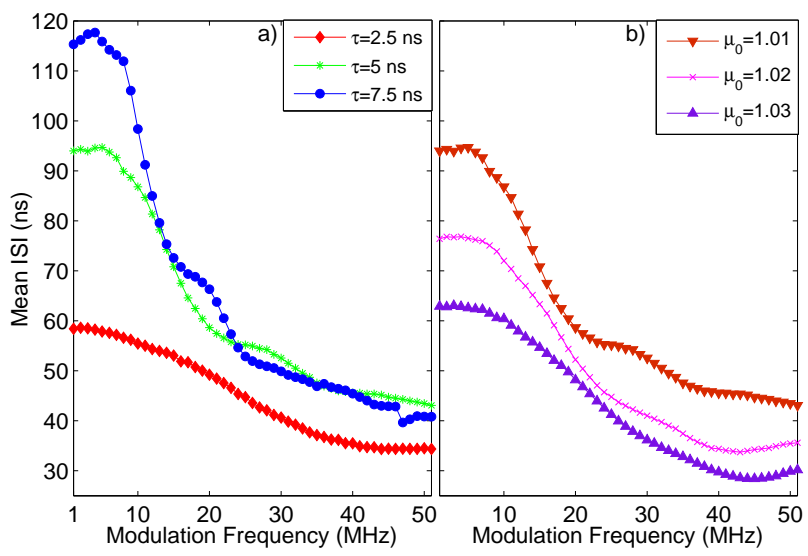

Fig. 4. a) Mean ISI from simulations as function of the modulation frequency for three different time delays. $\mu_{0}=1.01$. b) Mean ISI from simulations as function of the modulation frequency for three different DC current parameters, $\mu_{0} . \tau=5 \mathrm{~ns} . A_{\bmod }=1.6 \%$.

\section{Analysis of Spike Correlations via ORdinal SYMBOLIC ANALYSIS}

Although ordinal symbolic analysis does not take into account the exact duration of the inter-spike-intervals, it can capture subtle changes in time correlations among consecutive laser spikes, as the underlying correlations affect the probabilities of the ordinal patterns (OPs): if no correlations are present in the spike sequence, all OPs are equally probable; as there are $D$ ! possible OPs of dimension $D$, their expected probability is $1 / D$ !. Thus, if there are OPs whose probability is significantly different from $1 / D$ !, they unveil the existence of serial correlations in the timing of the laser spikes.

Figure 5 displays the results of the analysis of the experimental data: the probability of the pattern '210' is plotted for three delays and two modulation amplitudes. By analyzing the probability of this pattern, we investigate the existence of time correlations among 4 consecutive spikes. We chose this pattern because its probability is the one that differs the most from the $1 / 6$ value expected if no correlations are present in the spike sequence (i.e., if all the patterns are equally probable).

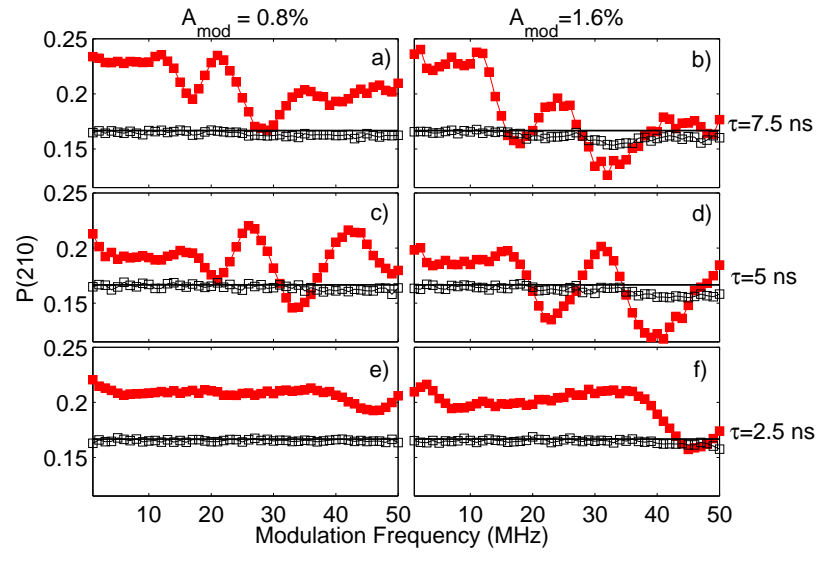

Fig. 5. Symbolic analysis of experimental ISI data: ' 210 ' probability against modulation frequency, for two modulation amplitudes and three time delays. a-b: $\tau=7.5 \mathrm{~ns}, I_{D C}=1.03 I_{t h}$. c-d: $\tau=5 \mathrm{~ns}, I_{D C}=1.024 I_{t h}$, threshold reduction: $7.1 \%$. e-f: $\tau=2.5 \mathrm{~ns}, I_{D C}=1.03 I_{t h}$. a,c,e: $A_{\text {mod }}=0.8 \%$ of $I_{t h}$. b,e,f: $A_{\text {mod }}=1.6 \%$ of $I_{t h}$. Full symbols: original data. Empty symbols: surrogate data.

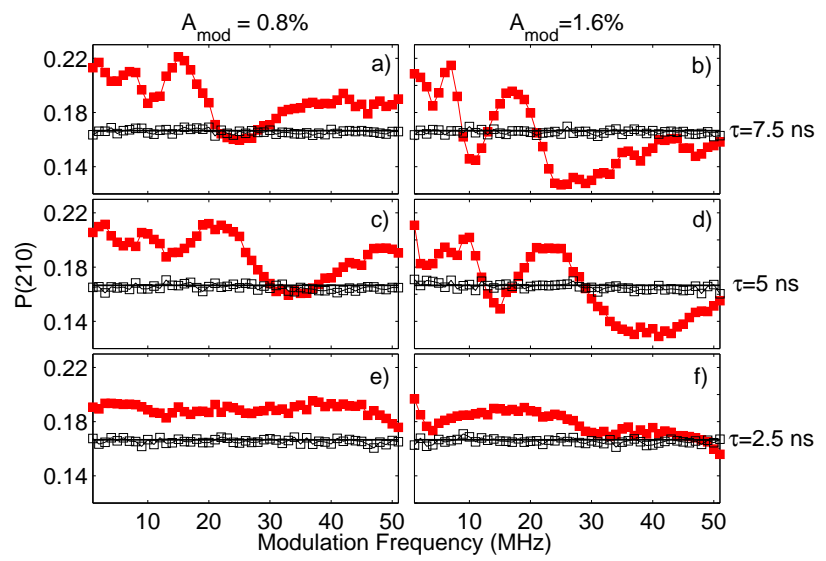

Fig. 6. Symbolic analysis of simulated ISI data: '210' probability against modulation frequency, for two modulation amplitudes and three time delays. $\mu_{0}=1.01$. a-b: $\tau=7.5$ ns. c-d: $\tau=5$ ns. e-f: $\tau=2.5$ ns. a,c,e: $A_{\bmod }=$ $0.8 \%$ of $I_{t h}$. b,e,f: $A_{\bmod }=1.6 \%$ of $I_{t h}$. Full symbols: original data. Empty symbols: surrogate data.

In order to demonstrate that the probability of this pattern indeed unveils the presence of spike correlations, in Fig. 5 we also plot in empty symbols the probability of ' 210 ' computed from surrogate data, i.e., when we shuffle the ISIs.

In panel 5a) there is a clear oscillation in the probability for intermediate frequencies. Observation of the changes in this oscillation pattern along the two columns (different amplitudes) and the three lines (different time delays), leads to the following conclusions: i) the increase of the modulation amplitude increases the differences between maxima and minima and moves the oscillation pattern to higher frequencies; ii) the decrease in the time delay decreases the differences between maxima and minima and moves the oscillation pattern to higher frequencies, in such a way that for $2.5 \mathrm{~ns}$ delay we can see only the first local minimum of the oscillation pattern.

In Fig. 6 we present the analysis of simulated data: the probability of ' 210 ' for original and surrogate data. A good agreement with the experimental results of Fig. 5 is observed. 


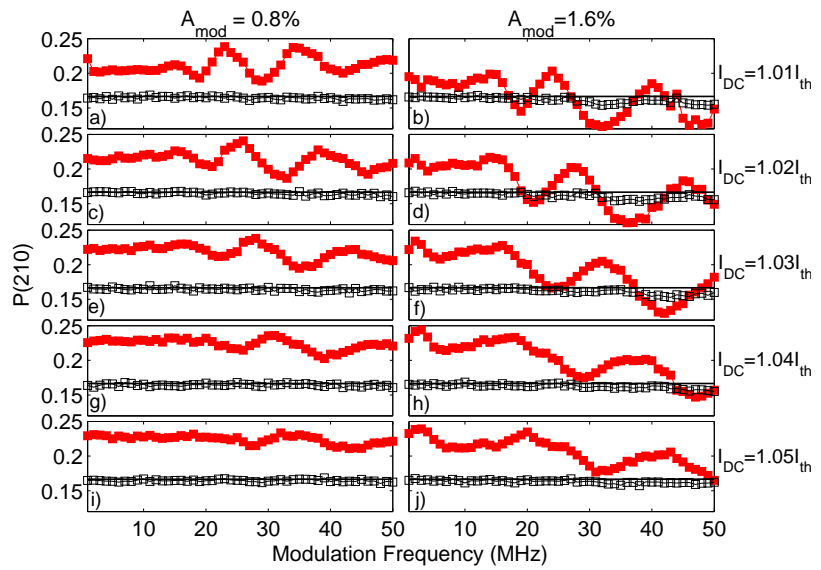

Fig. 7. Symbolic analysis of experimental ISI data: '210' probability against modulation frequency, for two modulation amplitudes and five $I_{D C} \cdot \tau=5$ ns. a-b: $I_{D C}=1.01 I_{t h}$. c-d: $I_{D C}=1.02 I_{t h}$. e-f: $I_{D C}=1.03 I_{t h}$. g-h: $I_{D C}=1.04 I_{t h}$. i-j: $I_{D C}=1.05 I_{t h}$. a,c,e,g,i: $A_{m o d}=0.8 \%$ of $I_{t h}$. $\mathrm{b}, \mathrm{d}, \mathrm{f}, \mathrm{h}, \mathrm{j}: A_{\bmod }=1.6 \%$ of $I_{t h}$. Full symbols: original data. Empty symbols: surrogate data.

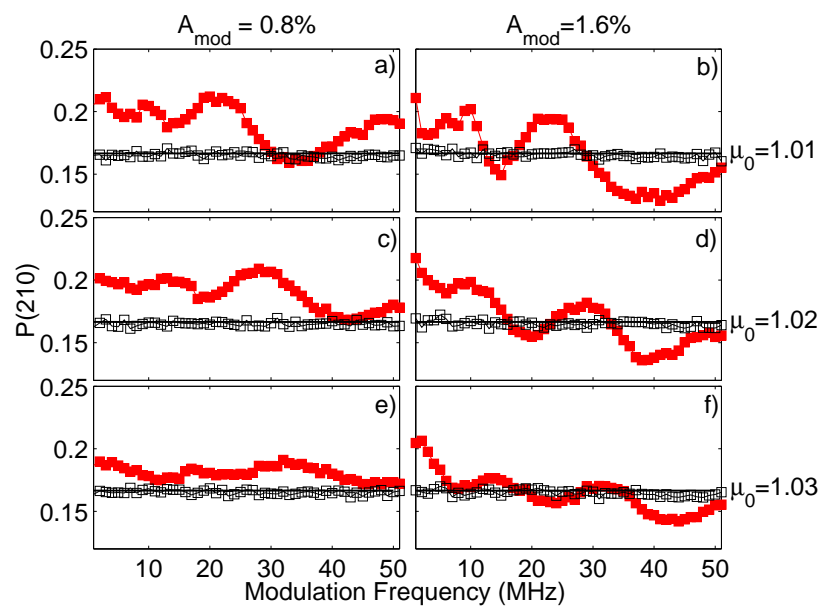

Fig. 8. Symbolic analysis of simulated ISI data: ' 210 ' probability against modulation frequency, for two modulation amplitudes and three values of $\mu_{0} . \tau=5$ ns. a-b: $\mu_{0}=1.01$. c-d: $\mu_{0}=1.02$. e-f: $\mu_{0}=1.03$. a,c,e: $A_{\text {mod }}=0.8 \%$. b,d,f: $A_{\text {mod }}=1.6 \%$. Full symbols: original data. Empty symbols: surrogate data.

A similar behavior is observed when the DC value of the injection current changes. In Fig. 7 we plot, for experimental ISIs, the probability of the ' 210 ' pattern for five different DC currents for the same modulation amplitudes and time delays as in Fig. 5. The variation of the oscillation pattern in the '210' probability when $I_{D C}$ increases is the same as in Fig. 5 when $\tau$ decreases, as in both cases the intrinsic (without modulation) spike rate increases. For the higher amplitude $(1.6 \%$, second column) maxima and minima are more pronounced, and they occur at higher modulation frequencies. For increasing injection current (from top to bottom) the probability curve becomes more flat, as the oscillation pattern moves to higher frequencies. These observations are the same for Fig. 8, where the probabilities for the pattern '210' are plotted for the simulated ISIs, for the same values of $\mu_{0}$ used in Fig. $4 \mathrm{~b}$.

From the observations above we can see that, as the dynamics becomes faster and the spike rate increases, the

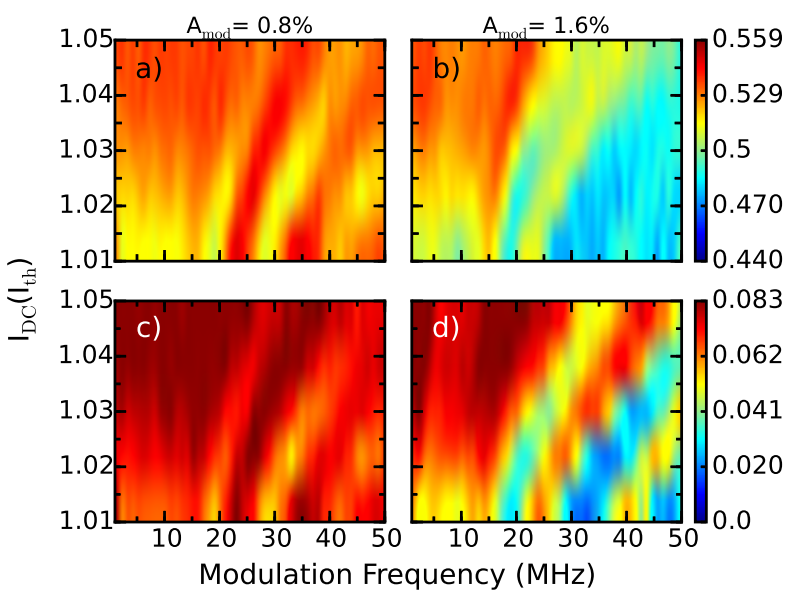

Fig. 9. Symbolic analysis of experimental ISI data. a,b: ' 10 ' OP probability for varying $I_{D C}$ and modulation current. c,d: '3210' OP probability for varying $I_{D C}$ and modulation frequency. a,c: $A_{\bmod }=0.8 \%$ of $I_{t h}$. b,d: $A_{\text {mod }}=1.6 \%$ of $I_{t h}$.

differences in the time correlations among 4 consecutive spikes for the different modulation frequencies fade away. Figure 9, that displays for experimental data the probabilities of the '10' (top row) and the '3210' (bottom row) patterns vs. the modulation frequency and $I_{D C}$, shows that it also occurs for the correlations among 3 and among 5 consecutive spikes. The same general trends observed for ' 210 ' can be seen here as the maxima and minima move to higher frequencies (see the color patterns shifting to the right and to the top) and the differences between maxima and minima diminish, as the injection current increases.

These results demonstrate that serial spike correlations tend to diminish as the spike rate of the unmodulated laser becomes faster (the laser spike rate increases either when the delay time is decreased, or when the pump current is increased).

\section{DISCUSSION}

As we have seen, ordinal analysis provides information about the presence of underlying serial correlations in the spike sequence, which complements the information that can be gained by applying traditional time-series analysis tools. Many studies of the modulated LFFs, using return maps, spectral measurements, etc., have been reported in the literature. For example, by using return maps, Giudici et al. [5] and Sukow and Gauthier [34] demonstrated experimentally that spikes occur preferentially at time intervals that are multiples of the modulation period. Lam et al. [33] proposed an explanation based on the adiabatic motion of the ellipse formed by the steady state solutions of the LK model, due to slow modulation. On the other hand, Mendez et al. [35] showed that the organization of the experimental periodic orbits was equivalent to that of the periodic solutions of a simple, low dimensional model proposed by Eguia et al. [50]. By analyzing the distribution of inter-spike-intervals, Buldú et al. [51] and Marino et al. [36] found evidence of stochastic resonance [52], as there is an optimal modulation frequency that maximizes the spike regularity. 
In our previous work [42] we used ordinal analysis to investigate how the correlations among several dropouts are affected by the modulation frequency and found that the minima and maxima of ' 210 ' OP probability were related to the noisy phase-locking of the spikes. Here we have focused on understanding how parameters that determine the natural spike rate (without modulation) affect this behavior. A crucial question remains that is: which physical mechanisms cause these correlations? While these are still unclear, because the same oscillations in the OP probabilities are seen in experimental and in numerical data, and they are clearly modified by model parameters (such as the pump current or the delay time), we speculate that the spike correlations are due to the specific organization of the trajectories in the systems phase space. The mechanisms responsible for spike correlations could also be related to the interplay of noise and modulation, in similar way as in stochastic resonance, where for an appropriated modulation frequency, the interplay of modulation and noise results in maximum spike regularity. Most importantly, these correlations could be generic features of periodically forced excitable systems: the observations of Feingold et al. [53] suggested that these systems can be described by circle maps, and several of us have shown [24] that a modified circle map adequately explains the correlations present in the LFF spikes, both, with and without modulation. In [24] it was shown that the OP probabilities (experimental observations and LK model simulations) display a well-defined, hierarchical and clustered structure, which is the same as that found in a modified circle map. Since the circle map describes many dynamical systems, including excitable ones, such correlations could also occur in other systems.

\section{CONCLUSION}

We have studied experimentally the dynamics of a directly modulated semiconductor laser with optical feedback in the LFF regime. Specifically, we studied how the external cavity length (i.e., the feedback delay time, $\tau$ ) and the DC value of the injection current, $I_{D C}$, affect the mean inter-spike-interval (ISI) and the spike correlations.

Although increasing the modulation frequency in general tends to decrease the mean ISI, the effect is non-monotonous and there are some oscillations. Moreover, the modulation frequency can have a strong or a small effect in the spike rate, depending on the parameters. Specifically, if the laser spike rate, without modulation, is slow (for large $\tau$ or for low $I_{D C}$ ), increasing the modulation frequency results in considerably faster spikes; on the contrary, if the spike rate is fast (for short $\tau$ or for high $I_{D C}$ ), the modulation frequency has only a small effect on the spike rate, and fast modulation is unable to produce much faster spikes.

By using symbolic ordinal analysis we also studied how the changes in the spike rate affect the correlations among several consecutive spikes. We calculated the probabilities of occurrence of the ordinal patterns (OPs) that represent increasingly close spikes: '3210', '210' and '10'. We used a clearly visible oscillation pattern in the OPs' probability, when it is plotted against the modulation frequency, to track the changes in the temporal correlations. We found an equivalent effect when decreasing the time delay or when increasing the DC value of the injection current, as the pattern moves to higher modulation frequency and the differences between maxima and minima fade out. As the intrinsic spiking dynamics becomes faster, the effects of the current modulation become less pronounced and the temporal correlations for the different modulation frequencies become all alike.

We also analyzed simulated spike sequences, using the Lang and Kobayashi model with typical parameters, and found a good qualitative agreement with the experimental observations.

Our observations are important for developing optical neurons that fully mimic biological ones, which encode the information about external input signals in the spike rate and in the spike timing. In other words, neuronal systems use sequences of correlated spikes for information encoding and processing and therefore, spike correlations should be carefully taken into consideration when designing optical neurons that mimic the behavior of biological neurons. Our results suggest that there is limited range of modulation frequencies that affect the spike rate and produce spike correlations: if the modulation is too fast, the spike correlations are washed out.

\section{ACKNOWLEDGMENT}

This work was supported by grants EOARD FA9550-14-10359, Ministerio de Economia y Competividad (Spain) and FEDER (European Union) project FIS2012-37655-C02-01, and the Marie Curie Initial Training Network NETT, FP7PEOPLE-2011-ITN 289146.

\section{REFERENCES}

[1] J. Ohtsubo, Semiconductor Lasers: Stability, Instabilities and Chaos, 3rd ed. Berlin Heidelberg, Germany: Springer-Verlag, 2013.

[2] D. Kane and K. A. Shore, editors, Unlocking Dynamical Diversity: Optical Feedback Effects on Semiconductor Lasers, West Sussex, England: Wiley, 2005.

[3] M. Fujiwara, K. Kubota, and R. Lang, "Lowfrequency intensity fluctuation in laser diodes with external optical feedback", Appl. Phys. Lett., vol.38, p. 217, 1981

[4] D. W. Sukow, J. R. Gardner, and D. J. Gauthier, "Statistics of powerdropout events in semiconductor lasers with time-delayed optical feedback," Phys. Rev. A., vol. 56, R3370, 1997.

[5] M. Giudici, C. Green, G. Giacomelli, U. Nespolo, and J. R. Tredicce, "Andronov bifurcation and excitability in semiconductor lasers with optical feedback," Phys. Rev. E, vol. 55, p. 6414, 1997.

[6] Y. Liu, P. Davis, and Y. Takiguchi, "Recovery process of low-frequency fluctuations in laser diodes with external optical feedback," Phys. Rev. E, vol. 60, pp.6595-6601, 1999.

[7] J. Mulet and C. R. Mirasso, "Numerical statistics of power dropouts based on the LangKobayashi model," Phys. Rev. E, vol. 59, p. 5400, 1999.

[8] M. Sciamanna, C. Masoller, N. B. Abraham, F. Rogister, P. Mégret, and M. Blondel, "Different regimes of low-frequency fluctuations in verticalcavity surface-emitting lasers," J. Opt. Soc. Am. B, vol. 20, p. 37, 2003.

[9] J. F. Martínez Avila, H. L. D. de S. Cavalcante, and J. R. Rios Leite, "Experimental deterministic coherent resonance," Phys. Rev. Lett., vol. 93, 144101, 2004.

[10] Y. Hong and K. A. Shore, "Statistical measures of the power dropout ratio in semiconductor lasers subject to optical feedback," Opt. Lett, vol. 30, p. 3332, 2005

[11] A. Torcini, S. Barland, G. Giacomelli, and F. Marin, "Low-frequency fluctuations in vertical cavity lasers: experiments versus LangKobayashi dynamics," Phys. Rev. A, vol. 74, 063801, 2006.

[12] J. Zamora-Munt, C. Masoller, and J. Garcia-Ojalvo, "Transient lowfrequency fluctuations in semiconductor lasers with optical feedback," Phys. Rev. A, vol. 81, 033820, 2010. 
[13] K. Hicke, X. Porte, and I. Fischer, "Characterizing the deterministic nature of individual power dropouts in semiconductor lasers subject to delayed feedback," Phys. Rev. E, vol. 88, 052904, 2013.

[14] Y. Takiguchi, Y. Liu, and J. Ohtsubo, "Low-frequency fluctuation induced by injection-current modulation in semiconductor lasers with optical feedback," Opt. Lett., vol. 23, pp. 1369-1371, 1998.

[15] B. Lindner, J. García-Ojalvo, A. Neiman and L. Schimansky-Geier, "Effects of noise in excitable systems," Phys. Rep., vol. 392, 321-424, 2004.

[16] E. Izhikevich, Dynamical Systems in Neuroscience: The Geometry of Excitability and Bursting, Cambridge MA, USA: The MIT Press, 2007.

[17] M. T. Hill, E. E. E. Frietman, H. de Waardt, G.-d. Khoe, and H. J. S. Dorren, All fiber-optic neural network using coupled SOA based ring lasers, IEEE Trans. Neural Netw., vol. 13, 1504-1513, 2002.

[18] A. Hurtado, I. D. Henning, and M. J. Adams, "Optical neuron using polarisation switching in a 1550nm-VCSEL," Opt. Express, vol. 18, 25170-25176, 2010.

[19] W. Coomans, L. Gelens, L. Mashal, S. Beri, J. Danckaert, and G. Van der Sande, "Solitary and coupled semiconductor ring lasers as optical spiking neurons," Phys. Rev. E, vol. 84, 036209, 2011.

[20] A. Hurtado, K. Schires, I. D. Henning, and M. J. Adams, "Investigation of vertical cavity surface emitting laser dynamics for neuromorphic photonic systems," Appl. Phys. Lett., vol. 100, 103703, 2012.

[21] M. Turconi, B. Garbin, M. Feyereisen, M. Giudici, and S. Barland, "Control of excitable pulses in an injection-locked semiconductor laser," Phys. Rev. E vol. 88, 022923, 2013.

[22] A. R. S. Romariz and K. H. Wagner, "Tunable vertical-cavity surfaceemitting laser with feedback to implement a pulsed neural model. 1. Principles and experimental demonstration," Appl. Opt., vol. 46, 47364745, 2007.

[23] A. R. S. Romariz and K. H. Wagner, "Tunable vertical-cavity surfaceemitting laser with feedback to implement a pulsed neural model. 2. High-frequency effects and optical coupling," Appl. Opt., vol. 46, 47464753, 2007.

[24] A. Aragoneses, S. Perrone, T. Sorrentino, M. C. Torrent, and C. Masoller, "Unveiling the complex organization of recurrent patterns in spiking dynamical systems," Sci. Rep., vol. 4, p. 4696, 2014.

[25] A. Aragoneses, T. Sorrentino, S. Perrone, D. J. Gauthier, M. C. Torrent, and C. Masoller, "Experimental and numerical study of the symbolic dynamics of a modulated external-cavity semiconductor laser," Opt. Express, vol. 22, pp. 4705-4713, 2014.

[26] E. C. Mos, J. J. L. Hoppenbrouwers, M. T. Hill, M. W. Blum, J. J. H B. Schleipen, and H. de Waardt, Optical neuron by use of a laser diode with injection seeding and external optical feedback, IEEE Trans. Neural Netw., vol. 11, 988-996, 2000.

[27] S. Barbay, R. Kuszelewicz, and A. M. Yacomotti, "Excitability in a semiconductor laser with saturable absorber," Opt. Lett., vol. 36, pp. 4476-4478, 2011.

[28] M. A. Nahmias, B. J. Shastri, A. N. Tait, and P. R. Prucnal, "A leaky integrate-and-fire laser neuron for ultrafast cognitive computing," IEEE J. Sel. Top. Quantum Electron., vol. 19, 1800212, 2013.

[29] F. Selmi, R. Braive, G. Breaudoin, I. Sagnes, R. Kuszelewicz, and S. Barbay, "Relative refractory period in an excitable semiconductor laser," Phys. Rev. Lett., vol. 112, 183902, 2014.

[30] B. J. Shastri, M. A. Nahmias, A. N. Tait, B. Wu, and P. R. Prucnal, "SIMPEL: Circuit model for photonic spike processing laser neurons," Opt. Express, vol. 23, 8029-8044, 2015.

[31] A. B. Neiman and D. F. Russell, "Models of stochastic biperiodic oscillations and extended serial correlations in electroreceptors of paddlefish," Phys. Rev. E, vol. 71, 061915, 2005.

[32] Y. Liu, N. Kikuchi, and J. Ohtsubo, "Controlling dynamical behavior of a semiconductor laser with external optical feedback," Phys. Rev. E, vol. 51, pp. R2697-R2700, 1995.

[33] W-S Lam, N. Parvez, and R. Roy, "Effect of spontaneous emission noise and modulation on semiconductor lasers near threshold with optical feedback," Int. J. of Modern Phys. B vol. 17, pp. 4123-4138, 2003.

[34] D. W. Sukow and D. J. Gauthier, "Entraining power-dropout events in an external-cavity semiconductor laser using weak modulation of the injection current," IEEE J. Quantum Electron., vol. 36, p. 175, 2000

[35] J. M. Mendez, R. Laje, M. Giudici, J. Aliaga, and G. B. Mindlin, "Dynamics of periodically forced semiconductor laser with optical feedback," Phys. Rev. E, vol. 63, 066218, 2001.

[36] F. Marino, M. Giudici, S. Barland, and S. Balle, "Experimental evidence of stochastic resonance in an excitable optical system," Phys. Rev. Lett., vol. 88, 040601, 2002.
[37] J. P. Toomey, D. M. Kane, M. W. Lee, and K. A. Shore, "Nonlinear dynamics of semiconductor lasers with feedback and modulation," Opt. Express vol. 18, pp. 16955-16972, 2010.

[38] C. M. Ticos, I. R. Andrei, M. L. Pascu, and M. Bulinski, "Experimental control of power dropouts by current modulation in a semiconductor laser with optical feedback," Phys. Scr., vol. 83, 055402, 2011.

[39] T. Schwalger, J. Tiana-Alsina, M. C. Torrent, J. García-Ojalvo, and B. Lindner, "Interspike-interval correlations induced by two-state switching in an excitable system," Europhys. Lett., vol. 99, 10004, 2012.

[40] S. Thorpe, A. Delorme, and R. Van Rullen, "Spike-based strategies for rapid processing," Neural Netw., vol. 14, pp. 715-725, 2001.

[41] D. Nikolić, P. Fries, and W. Singer, "Gamma oscillations: precise temporal coordination without a metronome," Trends in Cognitive Sciences, vol. 17, p. 54, 2013.

[42] T. Sorrentino, C. Quintero-Quiroz, A. Aragoneses, M. C. Torrent, and C. Masoller, "Effects of periodic forcing on the temporally correlated spikes of a semiconductor laser with feedback", Opt. Express, vol. 23, 5571-5581, 2015.

[43] R. Lang and K. Kobayashi, "External optical feedback effects on semiconductor injection laser properties," IEEE J. Quantum Electron., vol. $16,347,1980$.

[44] C. Bandt and B. Pompe, "Permutation entropy: a natural complexity measure for time series," Phys. Rev. Lett., vol. 88, p. 174102, 2002.

[45] J. Tiana-Alsina, M. C. Torrent, O. A. Rosso, C. Masoller, and J. Garcia-Ojalvo, "Quantifying the statistical complexity of low-frequency fluctuations in semiconductor lasers with optical feedback," Phys. Rev. A, vol. 82, 013189, 2010.

[46] M. C. Soriano, L. Zunino, O. A. Rosso, I. Fischer and C. R. Mirasso, "Time scales of a chaotic semiconductor laser with optical feedback under the lens of a permutation information analysis," IEEE J. Quantum Electron., vol. 47, 252-261, 2011.

[47] J. P. Toomey and D. M. Kane, "Mapping the dynamic complexity of a semiconductor laser with optical feedback using permutation entropy," Opt. Express, vol. 22, 1713-1725, 2014.

[48] J. P. Toomey, D. M. Kane, and T. Ackemann, "Complexity in pulsed nonlinear laser systems interrogated by permutation entropy," Opt. Express, vol. 22, 17840-17853, 2014.

[49] N. Li, W. Pan, S. Xang, Q. Zhao, L. Zang, and P. Mu, "Quantifying the complexity of the chaotic intensity of an external-cavity semiconductor laser via sample entropy," IEEE J. Quantum Electron., vol. 50, 766-774, 2014.

[50] M. C. Eguia, G. B. Mindlin, and M. Giudici, "Low-frequency fluctuations in semiconductor lasers with optical feedback are induced with noise," Phys. Rev. E, vol. 58, 2636-2639, 1998.

[51] J. M. Buldú, J. Garc'a-Ojalvo, C. R. Mirasso, and M. C. Torrent. "Stochastic entrainment of optical power dropouts," Phys. Rev. E, vol. 66, 021106, 2002.

[52] L. Gammaitoni, P. H'anggi, P. Jung, and F. Marchesoni, "Stochastic Resonance," Rev. Mod. Phys., vol. 70, 223-287, 1998.

[53] M. Feingold, D. L. Gonzalez, O. Piro, and H. Viturro, "Phase locking, period doubling, and chaotic phenomena in externally driven excitable systems," Phys. Rev. A, vol. 37, 4060-4063, 1988.

Taciano Sorrentino received the B.Sc. degree in physics and the M.Sc. degree in atomic and molecular physics, in 2008 and 2010, respectively, from the Universidade Federal da Paraíba, João Pessoa, PB, Brazil. In 2010 he joined the Universidade Federal Rural do Semi-Árido, Mossoró, RN, Brazil, as an assistant professor. He is currently pursuing the Ph.D. degree in computational and applied physics at the Departament de Física i Enginyeria Nuclear, Universitat Politècnica de Catalunya, Terrassa, Barcelona, Spain. His research interests include semiconductor lasers dynamics and applications.

C. Quintero-Quiroz received the B.Sc. degree in Physics and the M.Sc. degree in Fundamental Physics from the Universidad de Los Andes, Mérida, Venezuela in 2010 and 2013, respectively. He joined the Departament de Física i Enginyeria Nuclear in 2014 where he is currenly pursuing the Ph.D. degree in computational and applied physics. His current research interests include semiconductor lasers, nonlinear dynamics systems and neural inspired optical systems. 
M. C. Torrent received the B.S., M.S., and Ph.D. degrees in Physics from the University of Barcelona, Spain. In 1991, she joined the Department of Physics and Nuclear Engineering, Polytechnical University of Catalonia, Spain, as a member of the faculty. Her scientific interest have gradually changed from theoretical problems in statistical mechanics to applied physics. She has been working too in spatiotemporal dynamics of broad-area lasers. Her current research is on nonlinear dynamics of semiconductor lasers.

Cristina Masoller is Associated Professor with the Universitat Politècnica de Catalunya (UPC), Barcelona, Spain. She received the B.Sc. and M.Sc. degrees in physics from the Universidad de la Repblica (UDELAR), Montevideo, Uruguay, and the Ph.D. degree in physics from Bryn Mawr College, Bryn Mawr, PA, USA, in 1999. She was a Visiting Researcher with the University of Nice Sophia Antipolis, Nice, France, in 1999, the Universitat de les Illes Balears, Palma, Spain, from 2001 to 2002, Bangor University, Bangor, U.K., from 2002 to 2003, and an Associated Researcher with International Centre for Theoretical Physics (ICTP, Trieste, Italy), from 2003 to 2006. She has been a Program Committee Member of several international conferences, including CLEO/Europe and the International Semiconductor Laser Conference. She has co-organized six workshops on Laser Dynamics and Nonlinear Photonics (Uruguay 2003, 2005, 2007, 2009, 2011, and 2013), and a two-week School on Nonlinear Optics and Nanophotonics for Ph.D. students (ICTP-South American Institute for Fundamental Research, 2013). She has published over 120 articles. Her research interests include optical instabilities and chaos in semiconductor lasers, optical rogue waves, synchronization and stochastic phenomena, and high-dimensional dynamics. A main research goal has been exploiting stochastic and nonlinear phenomena for novel applications. 